\title{
Usefull Hearing Preservation Is Improved in Vestibular Schwannoma Patients Who Undergo Stereotactic Radiosurgery Before Further Hearing Deterioration Ensures
}

Hideyuki Kano ( $\nabla$ kanoh@upmc.edu )

University of Pittsburgh https://orcid.org/0000-0003-4881-951X

Akiyoshi Ogino

University of Pittsburgh

Hao Long

University of Pittsburgh

Stephen Johnson

University of Pittsburgh

Andrew Faramand

University of Pittsburgh

Ajay Niranjan

University of Pittsburgh

John C. Flickinger

University of Pittsburgh

L. Dade Lunsford

University of Pittsburgh

\section{Clinical Study}

Keywords: Gamma knife surgery, vestibular schwannoma, Gardner-Robertson grade, hearing preservation, pure tone average, speech discrimination score

Posted Date: February 9th, 2021

DOl: https://doi.org/10.21203/rs.3.rs-180869/v1

License: (c) (1) This work is licensed under a Creative Commons Attribution 4.0 International License.

Read Full License 
Version of Record: A version of this preprint was published at Journal of Neuro-Oncology on March 17th, 2021. See the published version at https://doi.org/10.1007/s11060-021-03726-6. 


\section{Abstract}

Introduction

The present study evaluates whether hearing deterioration during observation reduces serviceable hearing preservation rates after stereotactic radiosurgery (SRS) in vestibular schwannoma (VS) patients with useful hearing.

\section{Methods}

We retrospectively analyzed 1447 VS patients who underwent SRS between 1992 to 2017 . We identified 100 VS patients who had Grade I Gardner- Robertson (GR) hearing at initial diagnosis but were observed without surgery or SRS. We compared hearing after SRS in 67 patients who retained GR Grade I hearing from initial diagnosis to SRS (the hearing maintenance or HM group) to 33 patients whose hearing worsened from GR grade I to grade II (the hearing deterioration or HD group). We also investigated whether a decline in pure tone average (PTA) or speech discrimination score (SDS) before SRS affected hearing preservation after SRS.

Results

The serviceable hearing (GR I and II) preservation in $\mathrm{HM}$ patients was $80 \% \mathrm{~s}, 63 \%$, and $51 \%$ at 3,5 , and 10 years, respectively. The serviceable hearing preservation in $\mathrm{HD}$ patients was $40 \%, 33 \%$, and $20 \%$ at 3,5 , and 10 years, respectively. In multivariate analysis, younger age ( $<55$ years, $p=0.012)$ and HM during observation $(p<0.001)$ improved serviceable hearing preservation rates. Patients whose PTA increased $\geq 15 \mathrm{~dB}(p=0.011)$ or whose SDS declined $\geq 10 \%(p=0.024)$ had reduced serviceable hearing preservation rates.

\section{Conclusions}

Hearing deterioration during observation before SRS reduced long term hearing preservation rate in VS patients with GR grade I hearing at initial diagnosis. SRS before hearing deterioration was recommended for hearing preservation.

\section{Introduction}

Vestibular schwannomas (VS) generally are slow-growing tumors that arise from the vestibular portion of the eighth cranial nerve. The most common presenting symptom is ipsilateral hearing loss[1]. The wide spread use of Magnetic Resonance Imaging (MRI) has led to earlier diagnosis of often smaller volume VS, so that some patients have remarkably good hearing at diagnosis[2, 3]. For such patients observation or "wait and scan" is often recommended[4]. For patients with symptomatic larger volume VSs, microsurgical resection remains a potent intervention[4]. Stereotactic radiosurgery (SRS) for VS was first reported in 1971 by Leksell[5]. SRS using the Leksell Gamm Knife (GK) (AB Elekta) for small to medium size VSs has a resection free rate of over $95 \%$ in 10 years with an associated transient facial neuropathy 
rate of $1-5 \%$ and a serviceable hearing preservation rate of $43-68 \%$ at 5 years after SRS[6-9]. Early SRS after diagnosis improves hearing preservation[10,11]. Based on referral patterns and wait and scan recommendations from initial consultants some patients with serviceable hearing were observed and underwent SRS only after hearing deterioration or tumor progression was recognized. The aim of this study was to evaluate whether hearing deterioration during observation of VS patients with GR grade I affected hearing preservation after SRS.

\section{Methods}

\section{Patients population}

We retrospectively analyzed 1447 VS patients who underwent GK SRS at our center between 1992 and 2017. Patients who underwent SRS before1992 received higher margin doses (18 Gy-20 Gy) and were excluded from this study. We also excluded patients who had undergone prior resection, prior radiation therapy, or who had neurofibromatosis type 2. We identified 100 VS patients who had Gardner-Robertson (GR) grade[12] I hearing at initial diagnosis and were then were observed. Serviceable hearing was defined as GR grade I and II (PTA $\leq 50 \mathrm{~dB}$ and SDS $\geq 50 \%$ ). The flow diagram of this study population is shown in Fig.1. We compared long term hearing after SRS in 67 patients whose hearing remained GR grade I from initial diagnosis to SRS (hearing maintenance or HM group) with 33 patients whose hearing worsened from GR grade I to II at the time of SRS (hearing deterioration or HD group). We also evaluated whether hearing preservation was related to worsening PTA and SDS values during the observation. There were 64 males and 36 females. The median age was 55.2 years (range, 24-80 years). Seventyseven patients had tinnitus before SRS, and 41 patients had vertigo symptoms before SRS. Prior to SRS, no patient had facial neuropathy, but two patients had trigeminal neuralgia, and 5 patients had trigeminal neuropathy. Twenty-eight patients were classified as Koos grade[13] I at SRS, 30 as grade II, 23 as grade III, and 19 as grade IV. The median interval between initial diagnosis and SRS was 17.4 months (range, 3.1-190.2 months). Median follow-up after SRS was 4.4 years (range, 0.5-21.0 years). The detailed patient characteristics are shown in Table 1. This study was approved by the University of Pittsburgh Institutional Review Board (IRB 0404192).

\section{Radiosurgery Technique}

SRS was performed in a single procedure that began with stereotactic head frame application using local anesthesia supplemented by intravenous conscious sedation. Patients then underwent high-definition MRI and/or CT imaging studies as appropriate. SRS was performed using various models of the Leksell Gamma knife R (Model U, B, C, 4C, Perfexion and Icon (Elekta AB)). Dose planning was performed using various versions of the Leksell dose planning software (KULA or Leksell Gammaplan) [6, 14]. Radiation was delivered in a single session with typical margin dose of 12-13 Gy delivered to the tumor margin. The median margin dose was 12.5 Gy (range, 11.5-15.0 Gy). The median maximum dose was 25.0 Gy (range, 13.6-30.0 Gy). Tumor volume was defined as the calculated volume enclosed in treatment marginal dose at the time of SRS. The median target tumor volume was $0.60 \mathrm{~cm}^{3}$ (range, $0.06-11.0 \mathrm{~cm}^{3}$ ). 


\section{Patient follow-up}

After SRS, patients had imaging, hearing testing and clinical examination recommended at $0.5,1,2,4,8$, $12,16,20$ and 24 years. At any time when new symptoms were detected patients underwent a CT and/or MRI.

\section{Statistical analysis}

The data was analyzed using SPSS Statistics, version 25.0 (IBM, New York, USA). The relationship between HM and HD patients were analyzed statistically using Fischer's exact test and Mann-Whitney analysis as appropriate. Age, sex, tinnitus before SRS, SDS at diagnosis, target volume, margin dose, maximum dose, interval between diagnosis and SRS, and follow up time were not significantly different between these groups. We did find that HM patients reported less vertigo symptoms and had better PTA values than HD patients (Table 1). More HM patients had Koos grade I tumors compared to HD patients (Table 1). The detailed difference between HM and HD patients are shown in Table 1. Tumor enlargement after SRS was defined as a tumor volume that increased by $\geq 15 \%$. Kaplan-Meier plots for tumor control rates were created. Hearing deterioration was defined as a decline from GR grade I or II (serviceable) to III$\mathrm{V}$ (non-serviceable, SDS $<50 \%$ or PTA $>50 \mathrm{~dB}$ ). Kaplan-Meier plots for hearing preservation rates were created starting at the date of SRS. Univariate analysis was performed on the Kaplan-Meier method using a log-rank test. Multivariate analysis was performed using the Cox proportional hazards model. The suggested cutoff value for variables (age, PTA at diagnosis, SDS at diagnosis, target volume, margin dose, and interval between diagnosis and SRS) were determined by a Youden index based on receiver operating characteristic curve analysis[15]. $P$ value of $<0.05$ were considered statistically significant.

\section{Results}

\section{Tumor control}

The tumor control rate after SRS was $98.9 \%$ at 3 years, $95.3 \%$ at 5 years, and $92.2 \%$ at 10 years. In univariate analysis, there was no significant difference in tumor control rates between HM and HD patients $(p=0.535)$. Four patients had tumor progression during follow-up. Three patients were observed without additional treatment because the tumors had initial enlargement but then stabilized. These patients were one HD patient and two HM patients, but all three patients had hearing deterioration from 12 to 54 months after SRS. A single patient underwent additional SRS (margin dose $11.0 \mathrm{~Gy}$ ) at 4.5 years after initial SRS. This patient was HM patient, maintained useful hearing, and had no complications at 23 months after repeat SRS.

\section{Serviceable hearing preservation}

At the last follow up, 42 of $67 \mathrm{HM}$ patients maintained serviceable hearing, whereas 8 of $33 \mathrm{HD}$ patients had preserved serviceable hearing. The detailed comparison of GR hearing at diagnosis, SRS, and last follow-up are shown in Table 2. The overall serviceable hearing preservation rate was $65.9 \%$ at 3 years, 
$52.5 \%$ at 5 years and $40.5 \%$ at 10 years (Fig. $2 a$ ). The serviceable hearing preservation rates of HM patients were $79.9 \%$ at 3 years, $63.4 \%$ at 5 years, and $51.2 \%$ at 10 years. In contrast the serviceable hearing preservation rates of $\mathrm{HD}$ patients were $40.0 \%$ at 3 years, $32.7 \%$ at 5 years, and $19.6 \%$ at 10 years (Fig. 2b). In univariate analysis, younger age ( $<55$ years, $p=0.006)$, better PTA at diagnosis $(<20 \mathrm{~dB}$, $p=0.024)$, and HM status $(p<0.001)$ were associated with improved long-term serviceable hearing preservation rates. The following factors were not significantly associated with serviceable hearing preservation rates: sex $(p=0.119)$, tinnitus $(p=0.643)$, vertigo symptoms $(p=0.118)$, Koos grade (grade I \& II vs. III \& IV, $p=0.882)$, SDS at diagnosis ( $<94 \%$ vs. $\geq 94 \%, p=0.603)$, target tumor volume $\left(<0.6 \mathrm{~cm}^{3} v s . \geq 0.6\right.$ $\mathrm{cm}^{3}, \mathrm{p}=0.510$ ), margin dose ( $<13$ Gy vs. $\geq 13 \mathrm{~Gy}, \mathrm{p}=0.729$ ), and observation interval ( $<2$ years vs. $\geq 2$ years, $\mathrm{p}=0.706)$. In multivariate analysis, younger age ( $<55$ years, $\mathrm{p}=0.012, \mathrm{HR} 2.21,95 \% \mathrm{Cl} 1.19-4.11)$ and $\mathrm{HM}$ group (HM status, $p<0.001, \mathrm{HR} 2.85,95 \% \mathrm{Cl} 1.68-5.41$ ) were significantly associated with improved serviceable hearing preservation rates (Table 3 ).

\section{Does deterioration of the PTA or SDS during observation affect hearing preservation after SRS?}

During the observation interval (median interval 17.4 months) before SRS, the median PTA increased from $16.9 \mathrm{~dB}$ to $21.25 \mathrm{~dB}$ and the median SDS decreased from $96 \%$ to $92 \%$. The median increase in PTA was $6.85 \mathrm{~dB}$ and the median decrease in SDS was $4 \%$. In univariate analysis, patients with an increase in the PTA $\geq 15 \mathrm{~dB}$ during observation had reduced serviceable hearing preservation rates $(p=0.024)$ (Fig. $2 c$ ). Similarly, patients whose SDS decreased $\geqq 10 \%$ had reduced serviceable hearing preservation rates $(p=0.024)$ (Fig. 2d). GR grade I HM patients whose PTA worsened by $\geq 6 \mathrm{~dB}$ had significantly reduced GR grade I maintenance ( $p=0.005$ ) (Fig. 2e). Patients whose SDS decreased by $\geq 5 \%$ also had a reduced probability of maintaining GR grade I hearing ( $p=0.028)$ (Fig. 2f). Patients with a PTA at SRS of $<20 \mathrm{~dB}$ $(p=0.010)$ had a higher probability of maintaining GR grade I.

\section{Facial and trigeminal neuropathy}

In this series, 99\% maintained House-Brackman[16] (HB) grade I facial nerve function after SRS. One patient developed mild facial neuropathy (HB grade III) at 20.7 months after SRS. The Margin dose of this patient was $12.5 \mathrm{~Gy}$ and the tumor volume remained stable. Three patients developed transient facial spasms 8 to 38 months after SRS. No patient developed trigeminal neuropathy (defined as decrease in sensation, new pain or new paresthesia within ipsilateral trigeminal nerve distribution after SRS) in this series.

\section{Discussion}

Current management options for newly diagnosed VSs include observation, SRS, and surgical resection[4, 17]. In prior decades before the wide availability of MRI for screening of patients with asymmetric hearing loss, tinnitus, or disequilibrium symptoms, many VS tumors were not detected until the tumor resulted in major cranial nerve or neurological dysfunction. For symptomatic larger tumors with brain stem compression early complete resection became the most frequent intervention in patients without 
significant medical comorbidities. In patients with significant medical comorbidities subtotal resection followed by adjuvant SRS more recently has become an additional option[18-20]. In 1971, Leksell first reported the potential role of SRS for vestibular schwannomas (acoustic neuromas) [5]. In 1989, Kondziolka et al reported 162 consecutive VS patients who underwent Gamma knife SRS; they noted tumor control in $98 \%$ and unchanged hearing in $51 \%$ of patients [21]. In 2006, Pollock et al. reported a prospective cohort study comparing surgical resection with SRS for patients with small- to moderatesized VS[22]. They described no difference in tumor control, and described excellent cranial nerve preservation rates. After these and many other SRS reports emerged, further observation of smaller tumors rather than early surgical intervention was increasingly recommended.

Further observation was based on the premise that VS tend to grow slowly, and in any case outcomes from intervention are not worsened during an observational interval after the first confirmatory MRI[23, 24]. The present report examines the question of whether even mild hearing deterioration during an initial observation (or "wait and scan") interval leads to worse hearing outcomes in a series of patients who eventually proceed with SRS.

\section{Prior Reported Hearing Results in SRS Patients}

We reported that VS patients with serviceable hearing at the time of SRS had overall serviceable hearing preservation rates of $77.8 \%$ at 3 years, $68.8 \%$ at 5 years, and $51.8 \%$ at 10 years[14]. Akpinar et al. compared hearing outcomes in early SRS patients ( $\leq 2$ years after diagnosis) with late SRS hearing outcomes (>2 years after SRS). This report confirmed that earlier SRS resulted in better hearing preservation outcomes[11].

In the present study we evaluated hearing outcomes in patients who had normal or GR Grade 1 hearing at the time of initial diagnosis. In this retrospective study some patients maintained GR 1 hearing during the observation interval until SRS was performed. We compared those patients to a similar group of patients whose hearing had worsened during the observation interval. The multivariate analysis revealed that those without hearing worsening (HM Patients) and those younger than 55 years had significantly better useful hearing preservation rates after SRS. We and others have previously reported the beneficial effect of younger age in hearing preservation results after SRS $[9,14]$. The present study confirms that only those patients whose hearing does not worsen during observation have the best chance of long-term hearing preservation once SRS is performed. Patients whose hearing deteriorated during observation before SRS had worse hearing outcomes after SRS.

Regis et al. performed a study that evaluated intracanalicular VS patients who underwent early SRS or "wait and scan strategy" [10]. They found that tumor control and functional hearing preservation rates were higher in patients who underwent early SRS.

Many patients are now diagnosed by MRI performed for relatively mild or even non-specific symptoms such as asymmetric hearing loss, vague imbalance symptoms, vertigo, or tinnitus. If a small VS is found on MR, these patients are often referred to an otolaryngologist or neurosurgeon. After review of the 
hearing results, they may recommend a period of observation with a new scan in $6-12$ months. Patients may be told their tumor is benign and grows slowly. Some patients delay subsequent imaging if the symptoms remain mild and non-progressive. Referral for intervention by either surgery or radiosurgery may not occur for months or even years.

\section{What We Learned}

Based on our experience in more than 2000 VS patients over a 32 year interval, we suspected that earlier SRS might lead to better hearing preservation rates- and that hearing maintenance is an important outcome measure for many VS patients. We found that both PTA and SDS measurements are important predictors of hearing outcomes during such an observation interval. VS patients whose PTA increased $\geq 6$ $\mathrm{dB}$ or whose SDS decreased by $\geq 5 \%$ were less likely to retain GR grade I after SRS. Even more dramatic changes such as an PTA increase of $\geq 15 \mathrm{~dB}$ or a SDS decrease $\geq 10 \%$ during observation led to less serviceable hearing preservation. Of interest, Kirchman et al reported 10 years hearing outcomes in observed intracanalicular VS patients[25]. They found that the median PTA increased from $51 \mathrm{~dB}$ to 72 $\mathrm{dB}$ and median SDS decreased from $60 \%$ to $34 \%$ after follow-up of 9.5 years. In the current experience series, the median PTA increased from $16.9 \mathrm{~dB}$ to $21.3 \mathrm{~dB}$ at the time of SRS (median interval 17.4 months) and SDS decreased from $96 \%$ to $92 \%$.

\section{Study limitations}

This is a retrospective study, and lacks data related to tumor volume changes during the interval between first diagnosis and SRS. The HM group and HD group were not matched for PTA at diagnosis, presence of vertigo before SRS, and Koos grade. This study found that even mild hearing deterioration during the observation period before SRS significantly reduced the ability to maintain useful hearing after SRS.

\section{Conclusions}

To improve the long term maintenance of serviceable hearing in VS patients with Grade 1 hearing at diagnosis, we advocate SRS before further hearing deterioration is detected.

\section{Declarations}

Funding: None

Conflicts of interest / Competing interests: Dr. Lunsford is an AB Elekta stockholder.

Availability of data and material: All data pertaining to this research article are included within the manuscript as written.

Code availability: Not applicable to this work.

\section{Authors`contributions}


Conception and design: Hideyuki Kano, Akiyoshi Ogino. Acquisition of data: Akiyoshi Ogino, Hao Long, Stephen Johnson, Andrew Faramand. Analysis and interpretation of data: Akiyoshi Ogino. Drafting the article: Akiyoshi Ogino. Critically revising the article: Hideyuki Kano, L. Dade Lunsford. Reviewed submitted version of manuscript: All authors. Statistical analysis: Hideyuki Kano.

Administrative/technical/material support: L. Dade Lunsford, John C. Flickinger. Study supervision: Hideyuki Kano, L. Dade Lunsford, John C. Flickinger.

Ethics approval: All procedures performed in studies involving human participants were in accordance with the ethical standards of the institutional and/or national research committee and with the 1964 Helsinki declaration and its later amendments or comparable ethical standards.

Consent to participate: Informed consent was obtained from all individual participants included in the study.

Consent for publication: The authors affirm that human research participants provided informed consent for publication.

\section{References}

1. Matthies C, Samii M (1997) Management of 1000 vestibular schwannomas (acoustic neuromas): Clinical presentation. Neurosurgery 40:1-10. https://doi.org/10.1097/00006123-199701000-00001

2. Lin D, Hegarty JL, Fischbein NJ, Jackler RK (2005) The prevalence of "incidental" acoustic neuroma. Arch of Otolaryngol Head Neck Surg 131:241-244. https://doi.org/10.1001/archotol.131.3.241

3. Lunsford LD, Niranjan A, Flickinger JC, et al (2005) Radiosurgery of vestibular schwannomas: summary of experience in 829 cases. J Neurosurg 119 Suppl:195-199. https://doi.org/10.3171/jns.2005.102.s_supplement.0195

4. Goldbrunner R, Weller M, Regis J, et al (2019) EANO Guideline on the Diagnosis and Treatment of Vestibular Schwannoma. Neuro Oncol 22:31-45 https://doi.org/10.1093/neuonc/noz153

5. Leksell L (1971) A note on the treatment of acoustic tumours. Acta Chir Scand 137:763-765

6. Johnson S, Kano H, Faramand · Andrew, et al (2019) Long term results of primary radiosurgery for vestibular schwannomas. J Neurooncol 145:247-255. https://doi.org/10.1007/s11060-019-03290-0

7. Hasegawa T, Kida Y, Kato T, et al (2013) Long-term safety and efficacy of stereotactic radiosurgery for vestibular schwannomas: Evaluation of 440 patients more than 10 years after treatment with Gamma Knife surgery. J Neurosurg 118:557-565. https://doi.org/10.3171/2012.10.JNS12523

8. Lunsford LD, Niranjan A, Flickinger JC, et al (2005) Radiosurgery of vestibular schwannomas: Summary of experience in 829 cases. J Neurosurg 102:195-199. https://doi.org/10.3171/jns.2005.102.s_supplement.0195

9. Watanabe S, Yamamoto M, Kawabe T, et al (2016) Stereotactic radiosurgery for vestibular schwannomas: average 10-year follow-up results focusing on long-term hearing preservation. $J$ Neurosurg 125:64-72. https://doi.org/10.3171/2016.7.GKS161494 
10. Régis J, Carron R, Park MC, et al (2013) Wait-and-see strategy compared with proactive Gamma Knife surgery in patients with intracanalicular vestibular schwannomas: clinical article. J Neurosurg 119 Suppl:105-111. https://doi.org/10.3171/2010.8.GKS101058

11. Akpinar B, Mousavi SH, McDowell MM, et al (2016) Early radiosurgery improves hearing preservation in vestibular schwannoma patients with normal hearing at the time of diagnosis. Int J Radiat Oncol Biol Phys 95:729-734. https://doi.org/10.1016/j.ijrobp.2016.01.019

12. Gardner G, Robertson JH (1988) Hearing preservation in unilateral acoustic neuroma surgery. Ann Oto Rhinol Laryngol 97:55-66. https://doi.org/10.1177/000348948809700110

13. Koos WT, Day JD, Matula C, Levy DI (1998) Neurotopographic considerations in the microsurgical treatment of small acoustic neurinomas. J Neurosurg 88:506-512. https://doi.org/10.3171/jns.1998.88.3.0506

14. Johnson S, Kano H, Faramand A, et al (2020) Predicting hearing outcomes before primary radiosurgery for vestibular schwannomas. J Neurosurg 133:1235-1241. https://doi.org/10.3171/2019.5.jns182765

15. Youden WJ (1950) Index for rating diagnostic tests. Cancer 3:32-35. https://doi.org/10.1002/10970142(1950)3:1<32::AID-CNCR2820030106>3.0.C0;2-3

16. House JW, Brackmann DE (1985) Facial nerve grading system. Otolaryngol Head Neck Surg 93:146147. https://doi.org/10.1177/019459988509300202

17. Kondziolka D, Mousavi SH, Kano H, et al (2012) The newly diagnosed vestibular schwannoma: radiosurgery, resection, or observation? Neurosurg Focus 33:E8. https://doi.org/10.3171/2012.6.focus12192

18. Iwai Y, Yamanaka K, Ishiguro $T$ (2003) Surgery combined with radiosurgery of large acoustic neuromas. Surg Neurol 59:283-289. https://doi.org/10.1016/S0090-3019(03)00025-9

19. Starnoni D, Daniel RT, Tuleasca C, et al (2018) Systematic review and meta-analysis of the technique of subtotal resection and stereotactic radiosurgery for large vestibular schwannomas: a "nervecentered" approach. Neurosurg Focus 44:1-9. https://doi.org/10.3171/2017.12.FOCUS17669.

20. Van De Langenberg R, Hanssens PEJ, Van Overbeeke JJ, et al (2011) Management of large vestibular schwannoma. Part I. Planned subtotal resection followed by Gamma Knife surgery: Radiological and clinical aspects - Clinical article. J Neurosurg 115:875-884. https://doi.org/10.3171/2011.6.JNS101958

21. Kondziolka D, Lunsford LD, Mclaughlin MR, Flickinger JC (1998) Long-term outcomes after radiosurgery for acoustic neuromas. N Engl J Med 339:1426-1433. https://doi.org/10.1056/NEJM199811123392003

22. Pollock BE, Driscoll CLW, Foote RL, et al (2006) Patient outcomes after vestibular schwannoma management: A prospective comparison of microsurgical resection and stereotactic radiosurgery. Neurosurgery 59:77-83. https://doi.org/10.1227/01.NEU.0000219217.14930.14

23. Hillman TA, Chen DA, Quigley M, Arriaga MA (2010) Acoustic tumor observation and failure to followup. Otolaryngol Head Neck Surg 142:400-404. https://doi.org/10.1016/j.otohns.2009.10.047 
24. El Bakkouri W, Kania RE, Guichard JP, et al (2009) Conservative management of 386 cases of unilateral vestibular schwannoma: Tumor growth and consequences for treatment - Clinical article. $J$ Neurosurg 110:662-669. https://doi.org/10.3171/2007.5.16836

25. Kirchmann M, Karnov K, Hansen S, et al (2017) Ten-Year follow-up on tumor growth and hearing in patients observed with an intracanalicular vestibular schwannoma. Neurosurgery 80:49-56. https://doi.org/10.1227/NEU.0000000000001414

\section{Tables}

Table 1. Characteristics of 100 patients with vestibular schwannoma

\begin{tabular}{|lllll|}
\hline Characteristics & Total & HM group & HD group & p value \\
\hline Median age (years) & 55.2 & 53.6 & 59.4 & 0.104 \\
\hline Sex & & & & 0.121 \\
Male & 64 & 39 & 25 & \\
\hline Female & 36 & 28 & 8 & \\
\hline Tinnitus before SRS & 77 & 51 & 26 & 1.000 \\
\hline Vertigo/disequilibrium before SRS & 41 & 22 & 19 & 0.030 \\
\hline Koos grade & & & & 0.036 \\
\hline 1 & 28 & 22 & 6 & \\
\hline 2 & 30 & 16 & 14 & \\
\hline 3 & 23 & 19 & 4 & \\
\hline 4 & 19 & 10 & 9 & 0.165 \\
\hline Median PTA at diagnosis (dB) & 16.9 & 15.0 & 23.8 & $<0.001$ \\
\hline Median SDS at diagnosis (\%) & 96.0 & 96.0 & 96.0 & 0.615 \\
\hline Median target volume (cm ${ }^{3}$ ) & 0.60 & 0.58 & 0.78 & 0.312 \\
\hline Median margin dose (Gy) & 12.5 & 12.5 & 12.5 & 0.287 \\
\hline Median maximum dose (Gy) & 25.0 & 25.0 & 25.0 & 0.145 \\
\hline Median interval between diagnosis and SRS (months) & 17.4 & 16.8 & 19.1 & 0.245 \\
\hline Median follow-up after SRS (years) & 4.4 & 4.1 & 5.1 & 0.165 \\
\hline
\end{tabular}

Table 2. Comparison of Gardner-Robertson grade at diagnosis, SRS, and last follow-up 


\begin{tabular}{|llllll|}
\hline & Diagnosis & SRS & & \multicolumn{2}{l|}{ Last follow up } \\
\hline Group & GR grade & GR grade & No. of patients & GR grade & No. of patients \\
\hline HM Patients & I & I & 67 & I & 18 \\
& & & II & 24 \\
& & & III & 22 \\
& & & IV & 0 \\
HD Patients & I & & V & 3 \\
& & & II & I & 0 \\
& & & II & 8 \\
& & & III & 17 \\
& & & IV & 1 \\
& & & V & 7 \\
\hline
\end{tabular}

Table 3. Univariate and multivariate analysis for serviceable hearing status after radiosurgery 


\begin{tabular}{|c|c|c|c|c|}
\hline \multirow[t]{2}{*}{ Variable } & \multirow{2}{*}{$\begin{array}{l}\text { Univariate } \\
\mathrm{p} \text { value }\end{array}$} & \multicolumn{3}{|c|}{ Multivariate } \\
\hline & & value & $\mathrm{HR}$ & $95 \% \mathrm{Cl}$ \\
\hline Age (<55 vs. $\geq 55)$ & 0.006 & 0.012 & 2.21 & $\begin{array}{l}1.19- \\
4.11\end{array}$ \\
\hline Age (continuous) & 0.119 & NA & NA & NA \\
\hline Sex & 0.304 & NA & NA & NA \\
\hline Tinnitus before SRS (no vs. yes) & 0.643 & NA & NA & NA \\
\hline Vertigo/disequilibrium before SRS (no vs. yes) & 0.118 & 0.524 & NA & NA \\
\hline Koos grade (grade I \& II vs. III \& IV) & 0.882 & NA & NA & NA \\
\hline PTA at diagnosis (<20 dB vs. $\geq 20 \mathrm{~dB}$ ) & 0.024 & 0.252 & NA & NA \\
\hline PTA at diagnosis (continuous) & 0.025 & NA & NA & NA \\
\hline SDS at diagnosis ( $<94 \%$ vs. $\geq 94 \%)$ & 0.603 & NA & NA & NA \\
\hline SDS at diagnosis (continuous) & 0.835 & NA & NA & NA \\
\hline Target tumor volume $\left(<0.6 \mathrm{~cm}^{3}\right.$ vs. $\left.\geq 0.6 \mathrm{~cm}^{3}\right)$ & 0.510 & NA & NA & NA \\
\hline Target tumor volume (continuous) & 0.708 & NA & NA & NA \\
\hline HM group vs. HD group & $<0.001$ & $<0.001$ & 2.85 & $\begin{array}{l}1.68- \\
5.41\end{array}$ \\
\hline Margin dose (<13 Gy vs. $\geq 13$ Gy) & 0.729 & NA & NA & NA \\
\hline $\begin{array}{l}\text { Interval between diagnosis and SRS ( }<2 \text { years vs. } \geq 2 \\
\text { years) }\end{array}$ & 0.706 & NA & NA & NA \\
\hline
\end{tabular}

Figures 


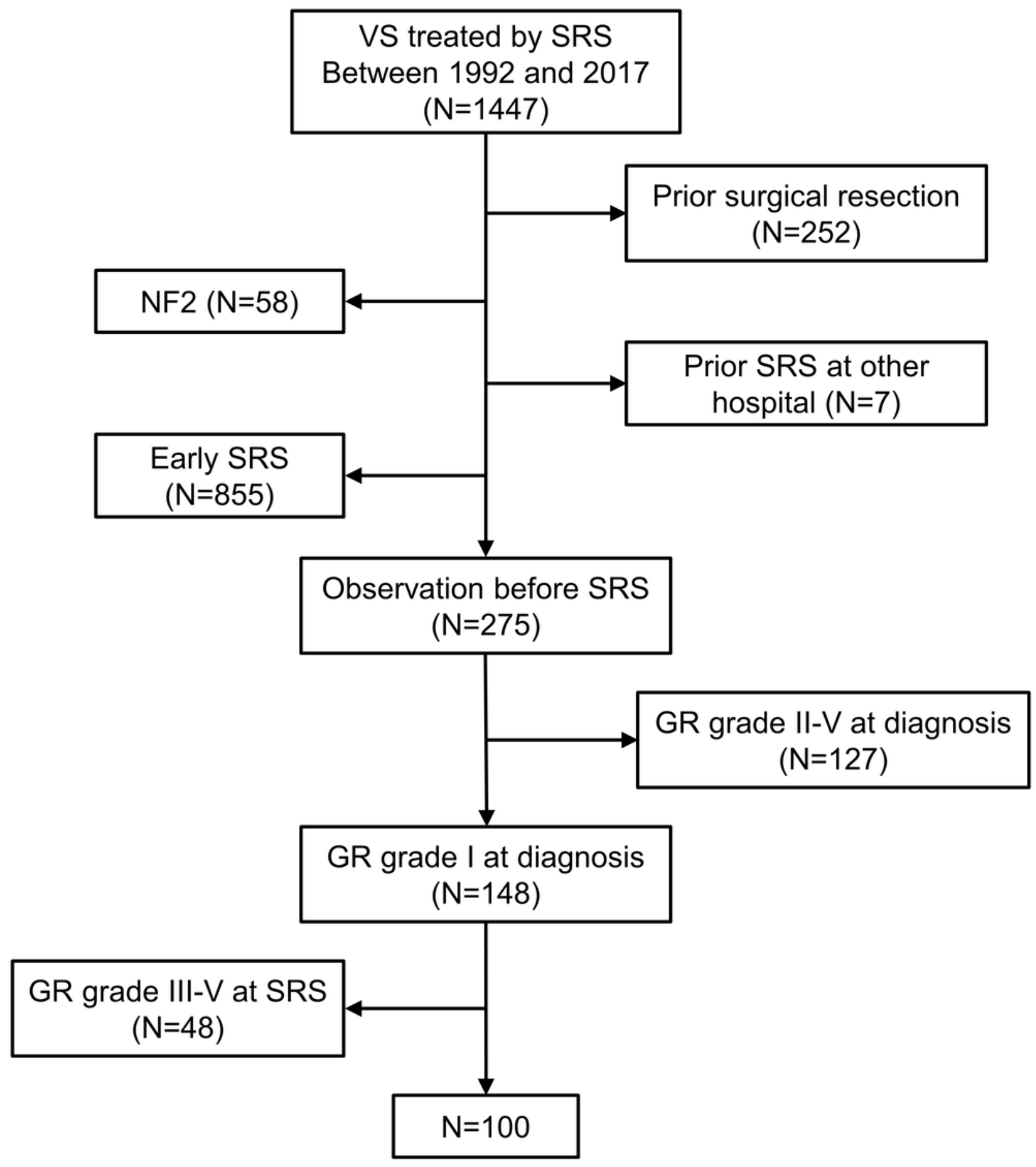

Figure 1

Flow diagram of this study population 



Figure 2

a: Kaplan-Meier plot demonstrating serviceable hearing preservation rate. b: Kaplan-Meier plot comparing serviceable hearing preservation rate of $\mathrm{HM}$ group and $\mathrm{HD}$ group. HM group has significantly better useful hearing preservation. c: Kaplan-Meier plot comparing serviceable hearing preservation rate of patients with increased PTA of $15 \mathrm{~dB}$ or more than $15 \mathrm{~dB}$ and less than $15 \mathrm{~dB}$. Increased PTA less than 15 dB was significantly associated with a higher serviceable hearing preservation rate $(p=0.011) d$ : Kaplan- 
Meier plot comparing serviceable hearing preservation rate of patients with decreased SDS of $10 \%$ or greater than $10 \%$ and less than $10 \%$. Decreased SDS less than $10 \%$ was significantly associated with a higher serviceable hearing preservation rate $(\mathrm{p}=0.024)$ e: Kaplan-Meier plot comparing probability of maintaining GR grade I of patients with increased PTA of $6 \mathrm{~dB}$ or greater than $6 \mathrm{~dB}$ and less than $6 \mathrm{~dB}$. Increased PTA less than $6 \mathrm{~dB}$ was significantly associated with a higher probability of maintaining GR grade I $(p=0.005)$ f: Kaplan-Meier plot comparing probability of maintaining GR grade I of patients with decreased SDS of $5 \%$ or greater than $5 \%$ and less than $5 \%$. Decreased SDS less than $5 \%$ was significantly associated with a higher probability of maintaining GR grade I $(p=0.028)$. 